\title{
An Empirical Examination of Factors Influencing the Intention to Use Physician Rating Websites
}

\author{
Nima Kordzadeh \\ Worcester Polytechnic Institute \\ nkordzadeh@wpi.edu
}

\begin{abstract}
Physician rating websites (PRWs) are social media platforms that enable patients to submit ratings and reviews of physicians. While numerous PRWs are available on the Internet and millions of physician reviews are posted on those websites, many people still do not use them when making clinical decisions. This study seeks to understand what factors impact intention to use PRWs. A sample of 109 students was employed. Each subject was randomly assigned to either RateMDs, Vitals, or Brigham and Women's Hospital's website. The subjects were asked to choose a primary care doctor based on the reviews posted on the assigned website and complete a survey accordingly. The regression analysis revealed that perceived credibility of reviewers and general use of online reviews influenced intention to use PRWs, whereas perceived integrity of website providers only moderated the relation between perceived credibility of reviewers and intention to use PRWs.
\end{abstract}

\section{Introduction}

In line with the growing adoption of social media and online review platforms in various sectors, patients in different countries such as United States [25, 27], Germany [11, 32], and China [21] use physician rating websites (PRWs) to review physicians and write comments about them. The reviews shared in this way can help other patients make more informed and judicious decisions on which doctors to visit. RateMDs, HealthGrades, and Vitals are among the most popular PRWs in the United States $[7,9]$. As of June 2018, over 2.6 million and 5 million reviews of healthcare professionals have been published on RateMDs [36] and Vitals [37], respectively.

Hospitals and clinics have also started offering their own physician rating services $[23,25]$. They send satisfaction surveys to their patients asking them about their care experience. The feedback including numeric ratings and narrative comments collected in this way are then published on the organization's website [25].
Cleveland Clinic, The University of Pittsburgh Medical Center (UPMC), and Brigham and Women's hospital in Boston are among the healthcare organizations that offer privately-administered physician review services. As of June 2018, for example, more than 440,000 reviews of healthcare professionals working at UPMC have been posted on their website [35].

Despite the growing number of PRWs and increasing number of physician reviews posted on those websites, a high percentage of the Internet users who are aware of such reviews do not use them when choosing a doctor $[14,20]$. Lack of trust in online reviews could be a major reason that many people are reluctant to use PRWs [20]. Prior studies have shown that trust in online reviews of products and services impact individuals' attitudes and decisions in nonhealthcare [6] and healthcare contexts [17]. Extant literature has also suggested that perceived credibility of reviewers and perceived integrity of rating website operators could play a role in forming people's trust perceptions and behavioral intentions [1, 22]. However, very few studies have actually examined these factors, in particular in the context of PRWs. This study seeks to fill this gap in the literature by analyzing the effects of perceived credibility of reviewers and perceived integrity of PRW providers on intention to use PRWs.

Perceived credibility of reviewers refers to the perceptions of Internet users on trustworthiness and genuine intentions of online reviewers [22]. Perceived integrity of PRW providers pertains to the perceptions of Internet users on honesty of PRW providers [5]. For example, reviewers who are believed to post fake or biased reviews may not be perceived to be credible. Also, websites whose operators are believed to remove negative reviews, manually add positive reviews, or intentionally manipulate patient reviews, could be perceived to lack integrity.

This study also introduces the concept of general online review use and seeks to understand whether those who generally use and rely on online consumer reviews when making decisions related to purchasing products, visiting restaurants, staying at hotels, etc. are 
more likely to use PRWs. Accordingly, the three research questions (RQ) addressed in this study are:

RQ1: How does perceived credibility of reviewers influence one's intention to use a PRW?

RQ2: How does perceived integrity of PRW providers influence one's intention to use a PRW?

RQ3: How does general online review use influence one's intention to use a PRW?

\section{Background}

Prior studies have examined different aspects of online physician reviews. Some researchers have investigated public awareness, adoption, and use of patient reviews of physicians. Hanauer, Zheng [19], for example, administered a survey to a nationally representative sample of parents and found that $74 \%$ of the respondents were aware of PRWs, whereas only $28 \%$ of the sampled parents had sought information and advice on those websites when choosing a primary care doctor for their children. Similarly, Terlutter, Bidmon [44] analyzed the knowledge and use of PRWs in Germany and found that $29.3 \%$ and $26.1 \%$ of the randomly selected 1006 patients were aware of, and had used, a PRW, respectively. The authors also reported that younger people, women, highly educated patients, and those with chronic diseases were more likely to use online physician reviews.

Other researchers have focused on the bias that inherently exists in patient reviews of physicians. Kadry, Chu [24] analyzed 10 frequently-visited PRWs, including RateMDs and HealthGrades, and found that the average ratings on the websites using 4point, 5-point, and 100-point scales were consistently around $77 \%$. The results of another study performed on online ratings of surgeons showed that the average rating of the 614 surgeons analyzed in that study was 4.4 out of 5 and $78.8 \%$ of the ratings were 4 or above [40]. Finally, Gao, McCullough [15] found that $45.80 \%$ of the physicians rated on RateMDs received the highest scores, whereas only $12 \%$ received the lowest scores. Collectively, the results of the studies under this research stream have demonstrated that patient ratings of physicians are mostly favorable to healthcare providers.

Prior studies, however, have rarely sought to understand what factors influence adoption and use of PRWs. Understanding the antecedents of using PRWs is important because it can help providers of commercial and hospital-affiliated PRWs improve the usability, performance, and adoption of their websites. Accordingly, the present study addresses this gap through investigating how trust perceptions and general online review use affect intention to use PRWs.

\section{Hypothesis development}

Reviewer credibility has become a major challenge in online communities and electronic word of mouth platforms. The main reason is that it is generally hard to confirm reviewers' identity and motivations for posting reviews on rating websites [22]. Firms, for example, could hire individuals and professional review-authoring companies to post promotional comments about their products and services and negative comments about competitors [28, 29, 31]. This fraudulent review generation process can ultimately make reviews unreliable, inaccurate, and biased [29, 48]. Users of rating websites who notice this bias may first try to assess the reviewers' credibility. To do so, website users may analyze a range of peripheral and central cues including reviewers' identifying information [12, 45], posting history [46], and online reputation [2], along with review characteristics such as the presence and types of textual errors in the reviews [8]. The results of such a subjective credibility assessment can impact one's attitudes toward the reviewed items, which in turn can influence one's purchase and use decisions [4].

In the context of online physician reviews, health information privacy policies, acts, and regulations prevent PRWs from collecting and sharing reviewers' posting history and identity information. This may make it hard for users of PRWs to assess the trustworthiness of each single reviewer and credibility of each review. Thus, people may use other information such as number of reviews [41], general skewness of ratings toward positive values [24], and general quality, bias, and informativeness of the comments about physicians posted on a PRW to assess the overall trustworthiness of reviewers on the website. Accordingly, it is expected that one's general credibility evaluations of reviewers determine one's willingness to use a PRW. We hypothesize:

H1. Perceived credibility of reviewers positively influences one's intention to use a PRW.

Consumer reviews are in general, a double-edged sword for organizations in various contexts [42]. While positive reviews about a company's products and services could improve their sales, negative reviews may significantly impact their brand reputation and success in the market [33]. In order to protect their reputation, many companies choose to respond to the consumer feedback and comments, in particular the unfavorable ones, posted on social networking sites such as Facebook and online review platforms such as Yelp and Amazon [42, 43]. Other companies adopt a censorship approach, which means 
they try to delete negative comments and feedback provided by consumers from the Internet as much as possible. Dekay [10] analyzed the official Facebook pages of 25 large corporations and found that 12 of them $(48 \%)$ actively engaged in the practice of deleting negative comments posted on their Facebook pages.

Although third-party consumer review platforms such as Yelp, TripAdvisor, and Amazon do not typically remove, or allow companies to remove negative consumer feedback, people may not be aware of this. Users of review websites may believe providers of those websites or companies whose products and services are rated on those websites can manipulate reviews and remove negative ones from those platforms. This belief could be stronger if users of a rating website notice that products and services have unusually received overly-positive ratings and comments [48]. This is the case for online physician reviews as prior studies have consistently shown that the reviews published on PRWs are highly skewed toward positive values and sentiments [15, 24].

In addition, commercial PRWs such as RateMDs, HealthGrades, and Vitals allow healthcare professionals to claim their profile on those websites. This will enable physicians to control their profile. For example, by paying $\$ 119$ a month, physicians can claim their profile on RateMDs, allowing them to control their profile picture and information, respond to reviews, hide ratings deemed to be suspicious, and feature a rating on that website [34]. If users of PRWs become aware of such features, they may become skeptical about the reliability and honesty of the reviews posted on those websites. The reason is that people may believe physicians, or website operators on behalf of physicians, are able to delete, hide, or manipulate reviews.

The physician ratings posted on hospitals' websites have also been shown to be highly skewed toward positive values and this skewness has been demonstrated to be stronger than corresponding skewness on commercial PRWs [25]. The average rating of UPMC doctors posted on that organization's website, for example, is 4.8, which could be perceived to be oddly high. The fact that hospitals control their own websites may imply it is possible for them to delete or manipulate reviews. This coupled with the positivity of reviews on hospital-affiliated websites may make people doubtful about the integrity and honesty of those websites in handling patient reviews. Thus, it is expected that these doubts and potential lack of trust could make people less inclined to use a PRW. We hypothesize:

H2. Perceived integrity of website providers positively influences one's intention to use a PRW.
Perceived credibility of reviewers and perceived integrity of website administrators may not only have direct effects on one's intention to use a PRW, but could interactively influence that intention. The reason is that those who trust the administrators and the vetting processes employed by a rating website could be confident that unreliable reviews and dishonest reviewers would be caught and handled properly by the website. This could lower one's concern about the role of untrustworthy reviewers in online reviews of physicians. Consequently, the impact of perceived credibility of reviewers on one's intention to use a PRWs could be reduced. In other words, there is a hypothetical interaction effect between perceived integrity of PRW providers and perceived credibility of reviewers such that the former negatively moderates the relation between the latter and intention to use PRWs. We hypothesize:

H3. Higher levels of perceived integrity of website providers weaken the impact of perceived credibility of reviewers on intention to use a PRW.

Consulting online reviews when collecting information about products and services and making purchase decisions can generally become a habit overtime. Those who develop such a habit may consistently use online reviews across different products and services. Accordingly, we define general online review use as the extent to which one uses online reviews when making decisions related to purchasing and using products and services in different categories. Prior studies in other contexts have taken a similar approach in conceptualizing and using constructs like general social networking sites use in the realm of online social networks [16, 39]. Given that patient reviews of physicians fall under the general category of online reviews of professional services, it is expected that higher levels of general online review use be associated with higher levels of intention to use PRWs. We hypothesize:

H4. People who generally use online reviews more often are more willing to use PRWs.

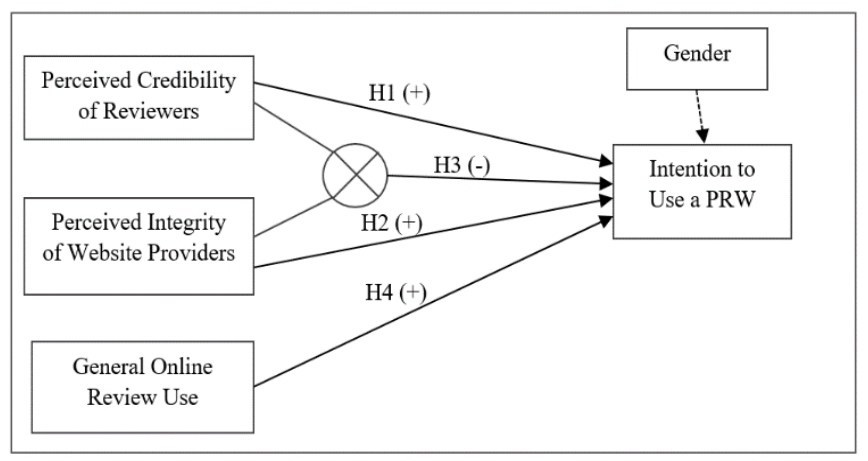

Figure 1: Research model and hypotheses 


\section{Method}

In order to test the hypotheses, we conducted an experimental study and used three actual websites including two commercial PRWs (RateMDs and Vitals) and one hospital website (Brigham and Women's Hospital). We used real rating platforms, and not experimentally-developed web pages, to make the study setting as realistic as possible and to examine whether the respondents would be willing to use those websites in the future. A convenience sample of undergraduate and graduate students was recruited from a private university located in the greater Boston area. Each respondent was randomly assigned to one of the three websites. The respondents were asked to assume they were going to choose a primary care doctor for themselves in the Boston area. They were provided with a link to the website they were assigned. The link would send them directly to the list of 'primary care doctors' working in 'Boston' who 'had reviews' on the assigned website. The respondents were asked to carefully examine the ratings and comments associated with each doctor, choose a doctor from the list accordingly, and then complete an online survey hosted on the Qualtrics website.
The survey instrument included measurement items associated with the four focal constructs (i.e., perceived credibility of reviewers, perceived integrity of website providers, general online review use, and intention to use a PRW). The items were adopted from the existing literature, where possible, and wording adjustments were made to them to make them fit into the context of the present study. The items were all measured on a 7-point Likert scale. Demographic items including education and gender were also included in the instrument. The measurement items are provided in Table1 1.

\section{Data analysis and results}

\subsection{Demographics}

In total, 109 students properly completed the survey, of which $63.3 \%$ (69/109) were undergraduate students and the rest were graduate students. Moreover, 59.6\% (65/109) of the respondents were female.

Table 1: Measurement items and PCA results

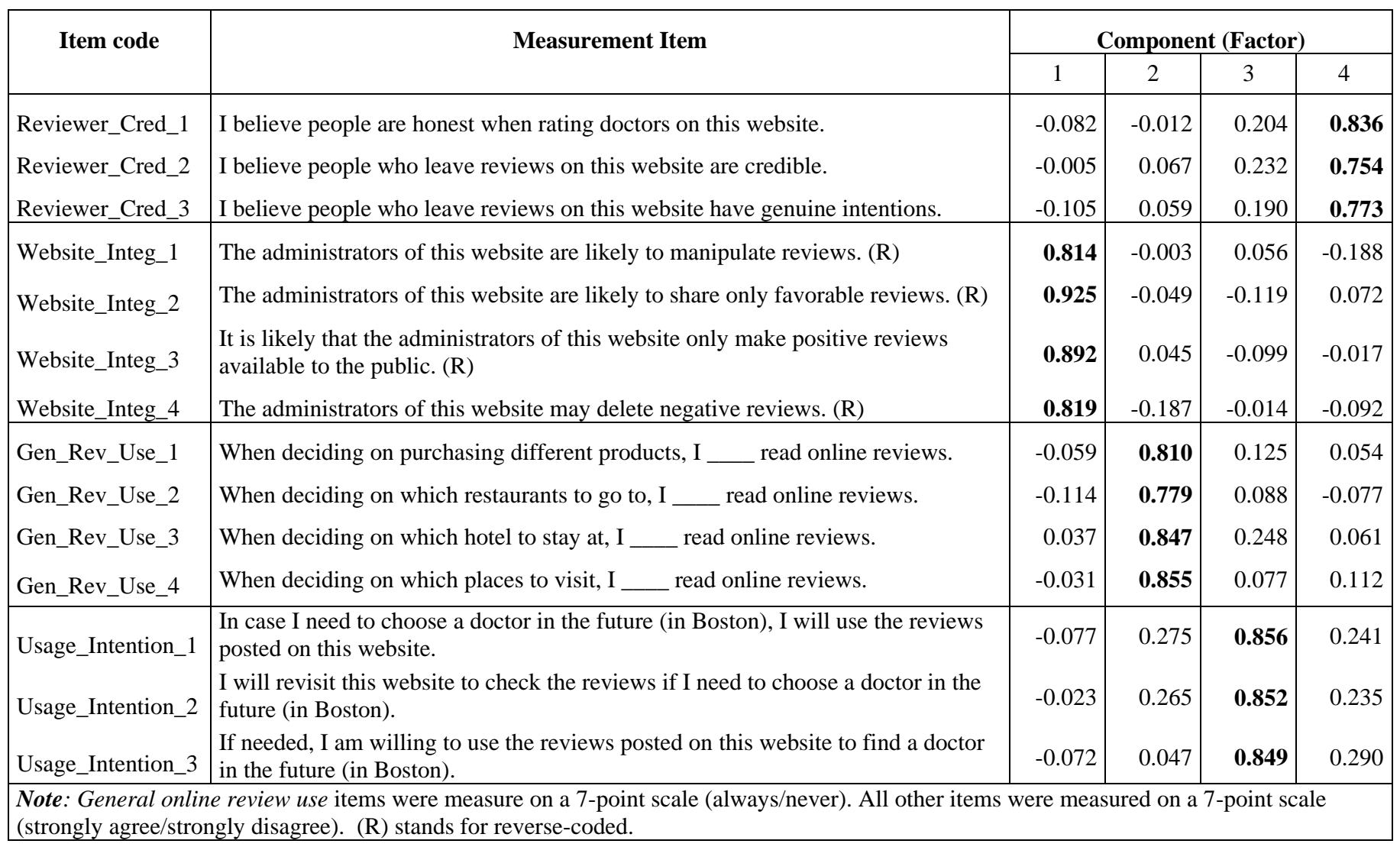




\subsection{Measurement validity and reliability}

In order to assess the convergent and discriminant validity of the scales, we first conducted a Principal Component Analysis (PCA) with Varimax rotation using SPSS 22.0. Four principal components were extracted explaining $75 \%$ of the total variance in the original items. The items adequately loaded on their corresponding constructs. The cross-loading values were all less than the acceptable threshold of 0.3 [18]. The final component structure is presented in Table 1 .

The descriptive statistics of the constructs as well as the inter-construct correlations are provided in Table 2. The off-diagonal items in that table display the Pearson correlation coefficients between the corresponding latent constructs. The diagonal items present the square root of average variance extracted (AVE) for the latent constructs. AVE was used to assess the convergent and discriminant validity of the scales [30]. Table 2 shows that all the AVEs are greater than 0.5 , supporting the convergent validity of the measurement scales [13]. Moreover, the square root of AVE for each construct exceeds the correlation between that construct and all other constructs, confirming the discriminant validity of the instrument [30]. Finally, we assessed the reliability of the measurement scales using Cronbach's alpha coefficients and composite reliability scores. The results provided in Table 2 showed that all the corresponding values were greater than the suggested threshold of 0.7 [38], supporting the reliability of the scales. In summary, the measures were demonstrated to be psychometrically appropriate.

Table 2: Inter-construct correlations and AVEs

\begin{tabular}{|c|c|c|c|c|c|c|c|c|c|}
\hline Construct & Mean & S.D. & $\begin{array}{l}\text { Cronbach's } \\
\text { alpha }(\alpha)\end{array}$ & $\begin{array}{l}\text { Composite } \\
\text { Reliability }\end{array}$ & AVE & 1 & 2 & 3 & 4 \\
\hline $\begin{array}{l}\text { 1. Reviewers' } \\
\text { Credibility }\end{array}$ & 4.61 & 0.84 & 0.76 & 0.863 & 0.673 & 0.820 & & & \\
\hline $\begin{array}{l}\text { 2. Website } \\
\text { Providers' } \\
\text { Integrity }\end{array}$ & 3.70 & 1.23 & 0.89 & 0.933 & 0.766 & 0.149 & 0.875 & & \\
\hline $\begin{array}{l}\text { 3. General Online } \\
\text { Review Use }\end{array}$ & 5.08 & 1.39 & 0.85 & 0.870 & 0.682 & 0.134 & 0.110 & 0.826 & \\
\hline $\begin{array}{l}\text { 4. Intention to } \\
\text { Use a PRW }\end{array}$ & 4.39 & 1.31 & 0.90 & 0.945 & 0.786 & $0.513 * *$ & 0.140 & $0.374 * *$ & 0.887 \\
\hline
\end{tabular}

\subsection{Hypothesis testing}

To test the hypotheses, we conducted a hierarchical moderated multiple regression (MMR) model, which has been shown to be an effective statistical technique to test main and interaction effects in a model $[3,26]$. The statistical model is provided in equation 1.

Equation 1: Intention_to_Use_PRW $=\beta_{0}+$ $\beta_{1}$.Gender $+\beta_{2}$. Reviewers_Credibility + $\beta_{3}$.Website_Providers_Integrity + $\beta_{4}$.General_Reivew_Use $+\beta_{5}$. Reviewers_Credibility $*$ Website_Providers_Integrity $+\varepsilon$

The model was performed in three steps: 1) only the control variable (gender) was included as a predictor , 2) the main effect variables (credibility of reviewers, integrity of website providers, and general online review use) were added to the model, and 3) the interaction term (between credibility of reviewers and integrity of website providers) was included in the model. The results of the regression analysis are presented in Table 3 and visualized in Figure 2. Columns a, b, and c of Table 3 are associated with the results of steps 1,2, and 3 of running the MMR model, respectively. The results demonstrated that among the hypothesized main effects, perceived credibility of reviewers and general online review use had significant relations with intention to use a PRW, supporting $\mathrm{H} 1$ and $\mathrm{H} 4$, respectively. Perceived integrity of website administrators, however, did not show any significant impact on the outcome variable, refuting H2. Moreover, the results supported the negative interaction effect between perceived credibility of reviewers and perceived integrity of website providers. Thus, H3 was supported. Finally, no significant relation was detected between gender and the outcome variable. In summary, three of the four hypothesized relations $(\mathrm{H} 1, \mathrm{H} 3, \mathrm{H} 4)$ were supported and one $(\mathrm{H} 2)$ was refuted. 
Table 3: Regression results

\begin{tabular}{|c|c|c|c|}
\hline Model & $\mathrm{a}$ & $\mathrm{b}$ & $\mathrm{c}$ \\
\hline Constant & $\begin{array}{l}4.432 * * * \\
(0.000)\end{array}$ & $\begin{array}{l}-0.497 \\
(0.465)\end{array}$ & $\begin{array}{l}-0.258 \\
(0.701)\end{array}$ \\
\hline \multicolumn{4}{|l|}{ Control Variable } \\
\hline $\begin{array}{l}\text { Gender } \\
(\text { Female }=1)\end{array}$ & $\begin{array}{l}-0.068 \\
(0.792)\end{array}$ & $\begin{array}{l}-0.255 \\
(0.228)\end{array}$ & $\begin{array}{l}-0.265 \\
(0.201)\end{array}$ \\
\hline \multicolumn{4}{|l|}{ Main Effects } \\
\hline $\begin{array}{l}\text { Perceived Credibility } \\
\text { of Reviewers }\end{array}$ & & $\begin{array}{l}0.747 * * * \\
(0.000)\end{array}$ & $\begin{array}{l}0.700 * * * \\
(0.000)\end{array}$ \\
\hline $\begin{array}{l}\text { Perceived Integrity of } \\
\text { Website Providers }\end{array}$ & & $\begin{array}{l}0.023 \\
(0.785)\end{array}$ & $\begin{array}{l}0.027 \\
(0.741)\end{array}$ \\
\hline $\begin{array}{l}\text { General Online } \\
\text { Review Use }\end{array}$ & & $\begin{array}{l}0.296 * * * \\
(0.000)\end{array}$ & $\begin{array}{l}0.299 * * * \\
(0.000)\end{array}$ \\
\hline \multicolumn{4}{|l|}{ Interaction Effect } \\
\hline $\begin{array}{l}\text { Perceived Credibility } \\
\text { of Reviewers * } \\
\text { Perceived Integrity of } \\
\text { Website Providers }\end{array}$ & & & $\begin{array}{l}-0.284 * \\
(0.022)\end{array}$ \\
\hline $\mathrm{R}^{2}$ & 001 & 0368 & 0400 \\
\hline Adjusted $\mathrm{R}^{2}$ & -0.009 & 0.344 & 0.371 \\
\hline$\Delta \mathrm{R}^{2}$ & 0.001 & 0.368 & 0.032 \\
\hline$\Delta \mathrm{F}$ & 0.070 & $20.178 * * *$ & $5.441^{*}$ \\
\hline \multicolumn{4}{|c|}{$\begin{array}{l}\text { Values in parentheses are } p \text {-values. } \\
* p<0.05, * * p<0.01, * * * p<0.001\end{array}$} \\
\hline
\end{tabular}

\section{Discussion}

Prior research in non-health contexts has shown that trust in online reviews can influence consumers' beliefs, attitudes, and behavioral intentions with regard to using online reviews and making purchase decisions [22, 47]. Prior studies have also suggested that uncertainties about trustworthiness of online review platforms as well as concerns regarding credibility of reviewers are two major factors that may make people willing or unwilling to use online consumer reviews [1]. Accordingly, this study mainly focused on examining the impact of trustworthiness of reviewers and integrity of PRW providers as well as general online review use on individuals' intention to use PRWs.

Our results demonstrated that individuals who generally used online reviews in different contexts and those who believed online reviewers were trustworthy were more willing to use a PRW. These results imply that people who visit a PRW may first assess the credibility of reviewers. Unlike the review platforms in other contexts (e.g., Yelp and TripAdvisor) that

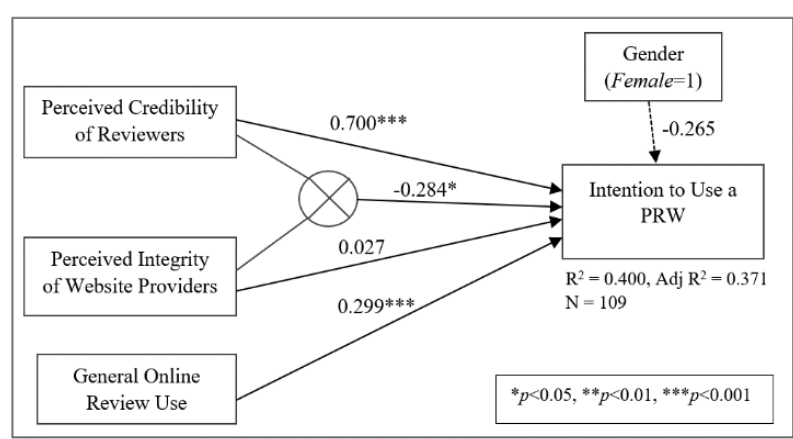

Figure 2: Hypothesis testing results

publish different reviewers' information such as name, review posting history, and number of followers [2], PRWs do not provide much information about reviewers due to privacy policies and regulations. Thus, users of PRWs may look for other cues such as consistency of positive ratings and comments posted for physicians on a website to evaluate the general trustworthiness of reviewers. Moreover, the results associated with general online review use supported the argument that using online reviews can become a habit, which may influence one's intention to use reviews of products and services in different contexts including healthcare.

Our findings also supported the hypothesized negative interaction effect between perceived credibility of reviewers and perceived integrity of PRW providers. Accordingly, if one believes a PRW is honest, perceived credibility of reviewers on that website may not impact users' willingness to revisit it as much as when one believes the website lacks integrity. Interestingly, the hypothesized direct relation between perceived integrity of PRW providers and intention to use such websites was not supported. A plausible explanation for this non-significant result is that people may believe even if PRW administrators delete negative reviews or manipulate some comments, such additions and manipulations are small in scale and may not make a considerable impact on the usability of PRWs.

A practical implication of the results for providers of commercial PRWs and healthcare organizations' websites is that credibility of reviewers is a critical factor in users' minds that determines usability of such websites. Therefore, providers of physician rating platforms and services should employ mechanisms and measures to ensure that individuals who post reviews about physicians are credible and hence, the published reviews are reliable and truthful. Once this is ensured, it should be properly and effectively communicated to the users of the rating websites to mitigate users' concerns regarding trustworthiness of reviewers. From a theoretical standpoint, our results 
enhanced understanding of trust in online reviews in the context of healthcare. Additionally, a new constructs named general use of online reviews was conceptualized and operationalized in this study, which can be used in future research.

\section{Limitations and future research}

This study has limitations. First, a student sample was recruited. Given the potential limitations of student samples [26], future studies can confirm and extend the results of this study by collecting data from non-student subjects. Moreover, only three websites were used in this research. Future studies can include more websites or develop experimental web pages to control for website-specific confounding factors and assess individuals' perceptions, attitudes, and intentions of using PRWs in lab-based experimental settings. Additionally, researchers in future studies can examine antecedents of perceived trustworthiness of reviewers and perceived integrity of PRW providers to better understand what characteristics of PRWs determine users' trust in those websites and what aspects of online reviews of physicians are assessed by users of PRWs to ensure the credibility of reviewers.

\section{Conclusion}

PRWs provide opinion-sharing platforms helping patients make more informed clinical decisions. However, different factors may discourage people from using such websites. This study aimed to understand three of those factors. The results highlighted the fact that users of online reviews are concerned about the credibility and trustworthiness of the sources of reviews. Additionally, the results suggest that perceptions of integrity and honesty of PRW providers negatively moderate the relation between perceived credibility of reviewers and intention to use a PRW. Thus, providers of commercial and hospital-affiliated PRWs should ensure their users that website providers do not systematically delete or manipulate reviews. PRW providers should also make sure only verified and credible reviewers rate physicians.

\section{References}

[1] J.K. Ayeh, N. Au, and R. Law. 2013. "“Do we believe in TripAdvisor?" Examining credibility perceptions and online travelers' attitude toward using user-generated content". Journal of Travel Research, 52(4), pp. 437-452.

[2] S. Banerjee, S. Bhattacharyya, and I. Bose. 2017. "Whose online reviews to trust? Understanding reviewer trustworthiness and its impact on business". Decision Support Systems, 96, pp. 17-26.

[3] T.A. Carte and C.J. Russell. 2003. "In pursuit of moderation: Nine common errors and their solutions". MIS Quarterly, pp. 479-501.

[4] C.M.-Y. Cheung, C.-L. Sia, and K.K. Kuan. 2012. "Is this review believable? A study of factors affecting the credibility of online consumer reviews from an ELM perspective". Journal of the Association for Information Systems, 13(8), pp. 618.

[5] C.M. Cheung and M.K. Lee. Trust in internet shopping: instrumental development and validation through classical and modern approaches, in Advanced Topics in Global Information Management, Volume 1. 2002, IGI Global. p. 25-41.

[6] C.M. Cheung and D.R. Thadani. 2012. "The impact of electronic word-of-mouth communication: A literature analysis and integrative model". Decision Support Systems, 54(1), pp. 461-470.

[7] M. Cloney, B. Hopkins, N. Shlobin, and N.S. Dahdaleh. 2018. "Online Ratings of Neurosurgeons: An Examination of Web Data and its Implications". Neurosurgery.

[8] D. Cox, J.G. Cox, and A.D. Cox. 2017. "To Err is human? How typographical and orthographical errors affect perceptions of online reviewers". Computers in Human Behavior, 75, pp. 245-253.

[9] T.J. Daskivich, J. Houman, G. Fuller, J.T. Black, H.L. Kim, and B. Spiegel. 2017. "Online physician ratings fail to predict actual performance on measures of quality, value, and peer review". Journal of the American Medical Informatics Association.

[10] S.H. Dekay. 2012. "How large companies react to negative Facebook comments". Corporate

Communications: An International Journal, 17(3), pp. 289299.

[11] M. Emmert, F. Halling, and F. Meier. 2015. "Evaluations of dentists on a German physician rating Website: an analysis of the ratings". Journal of Medical Internet Research, 17(1).

[12] C. Forman, A. Ghose, and B. Wiesenfeld. 2008. "Examining the relationship between reviews and sales: The role of reviewer identity disclosure in electronic markets". Information Systems Research, 19(3), pp. 291313.

[13] C. Fornell and D.F. Larcker. 1981. "Evaluating structural equation models with unobservable variables and measurement error". Journal of Marketing Research, pp. 39-50.

[14] S. Fox. The Social Life of Health Information, 2011. 2011 [Access Date: June 2018]; 
http://www.pewinternet.org/2011/05/12/the-social-life-ofhealth-information-2011/.

[15] G.G. Gao, J.S. McCullough, R. Agarwal, and A.K. Jha. 2012. "A changing landscape of physician quality reporting: analysis of patients' online ratings of their physicians over a 5-year period". Journal of Medical Internet Research, 14(1).

[16] H. Gil de Zúñiga, N. Jung, and S. Valenzuela. 2012. "Social media use for news and individuals' social capital, civic engagement and political participation". Journal of Computer-Mediated Communication, 17(3), pp. 319-336.

[17] S. Grabner-Kräuter and M.K. Waiguny. 2015. "Insights into the impact of online physician reviews on patients' decision making: randomized experiment". Journal of Medical Internet Research, 17(4).

[18] J. Hair, R. Anderson, R. Tatham, and W. Black. 1987. "Multivariate Data Analysis with Readings Macmillan". New Work.

[19] D.A. Hanauer, K. Zheng, D.C. Singer, A. Gebremariam, and M.M. Davis. 2014. "Parental awareness and use of online physician rating sites". Pediatrics, pp. peds. 2014-0681.

[20] D.A. Hanauer, K. Zheng, D.C. Singer, A. Gebremariam, and M.M. Davis. 2014. "Public awareness, perception, and use of online physician rating sites". Journal of the American Medical Informatics Association, 311(7), pp. 734-735.

[21] H. Hao. 2015. "The development of online doctor reviews in China: an analysis of the largest online doctor review website in China". Journal of Medical Internet Research, 17(6).

[22] M.L. Jensen, J.M. Averbeck, Z. Zhang, and K.B. Wright. 2013. "Credibility of anonymous online product reviews: A language expectancy perspective". Journal of Management Information Systems, 30(1), pp. 293-324.

[23] A.K. Jha. 2015. "Health care providers should publish physician ratings". Harvard Business Review.

[24] B. Kadry, L.F. Chu, B. Kadry, D. Gammas, and A. Macario. 2011. "Analysis of 4999 online physician ratings indicates that most patients give physicians a favorable rating". Journal of Medical Internet Research, 13(4).

[25] N. Kordzadeh. 2018. "Physician Ratings Published on Healthcare Organizations' Websites: Are They Biased?".

[26] N. Kordzadeh and J. Warren. 2017. "Communicating Personal Health Information in Virtual Health Communities: An Integration of Privacy Calculus Model and Affective Commitment". Journal of the Association for Information Systems, 18(1).

[27] T. Lagu, K. Metayer, M. Moran, L. Ortiz, A. Priya, S.L. Goff, and P.K. Lindenauer. 2017. "Website characteristics and physician reviews on commercial physician-rating websites". The Journal of the American Medical Association, 317(7), pp. 766-768.

[28] X. Li and L.M. Hitt. 2008. "Self-selection and information role of online product reviews". Information Systems Research, 19(4), pp. 456-474.

[29] M. Luca and G. Zervas. 2016. "Fake it till you make it: Reputation, competition, and Yelp review fraud". Management Science, 62(12), pp. 3412-3427.

[30] S.B. MacKenzie, P.M. Podsakoff, and N.P. Podsakoff. 2011. "Construct measurement and validation procedures in MIS and behavioral research: Integrating new and existing techniques". MIS Quarterly, 35(2), pp. 293-334.

[31] D. Mayzlin, Y. Dover, and J. Chevalier. 2014. "Promotional reviews: An empirical investigation of online review manipulation". American Economic Review, 104(8), pp. 2421-55.

[32] S. McLennan, D. Strech, A. Meyer, and H. Kahrass. 2017. "Public Awareness and Use of German Physician Ratings Websites: Cross-Sectional Survey of Four North German Cities". Journal of Medical Internet Research, 19(11).

[33] S.M. Mudambi and D. Schuff. 2010. "Research note: What makes a helpful online review? A study of customer reviews on Amazon. com". MIS Quarterly, pp. 185-200.

[34] n.d. Claim Your Profile: Select A Plan. 2018 [cited 2018; Available from:

https://www.ratemds.com/plans/1966162/.

[35] n.d. Find A Doctor. 2018 [Access Date: June 2018]; http://findadoc.upmc.com/FindADocSearch.aspx.

[36] n.d. RateMDs. 2018 [Access Date: June 2018]; https://www.ratemds.com.

[37] n.d. Vitals/about. 2018 [Access Date: June 2018]; https://www.vitals.com/about/patients.

[38] J. Nunnally. 1978. "Psychometric theory (2nd edit.) mcgraw-hill". Hillsdale, NJ.

[39] H.J. Oh, E. Ozkaya, and R. LaRose. 2014. "How does online social networking enhance life satisfaction? The relationships among online supportive interaction, affect, perceived social support, sense of community, and life satisfaction". Computers in Human Behavior, 30, pp. 6978.

[40] K. Okike, T.K. Peter-Bibb, K.C. Xie, and O.N. Okike. 2016. "Association between physician online rating and quality of care". Journal of Medical Internet Research, 18(12).

[41] D.-H. Park, J. Lee, and I. Han. 2007. "The effect of on-line consumer reviews on consumer purchasing 
intention: The moderating role of involvement". International Journal of Electronic Commerce, 11(4), pp. 125-148.

[42] M.D. Sotiriadis and C. Van Zyl. 2013. "Electronic word-of-mouth and online reviews in tourism services: the use of twitter by tourists". Electronic Commerce Research, 13(1), pp. 103-124.

[43] B.A. Sparks and G.L. Bradley. 2017. "A "Triple A" typology of responding to negative consumer-generated online reviews". Journal of Hospitality \& Tourism Research, 41(6), pp. 719-745.

[44] R. Terlutter, S. Bidmon, and J. Röttl. 2014. "Who uses physician-rating websites? Differences in

sociodemographic variables, psychographic variables, and health status of users and nonusers of physician-rating websites". Journal of Medical Internet Research, 16(3).
[45] H.J. Xie, L. Miao, P.-J. Kuo, and B.-Y. Lee. 2011.

"Consumers' responses to ambivalent online hotel reviews: The role of perceived source credibility and pre-decisional disposition". International Journal of Hospitality Management, 30(1), pp. 178-183.

[46] D. Zhang, L. Zhou, J.L. Kehoe, and I.Y. Kilic. 2016. "What online reviewer behaviors really matter? Effects of verbal and nonverbal behaviors on detection of fake online reviews". Journal of Management Information Systems, 33(2), pp. 456-481.

[47] K.Z. Zhang, S.J. Zhao, C.M. Cheung, and M.K. Lee. 2014. "Examining the influence of online reviews on consumers' decision-making: A heuristic-systematic model". Decision Support Systems, 67, pp. 78-89.

[48] M. Zhuang, G. Cui, and L. Peng. 2018. "Manufactured opinions: The effect of manipulating online product reviews". Journal of Business Research, 87, pp. 24-35. 